\title{
Differential proteomics analysis of liver failure in peripheral blood mononuclear cells using isobaric tags for relative and absolute quantitation
}

\author{
HUA LIN $^{1 *}$, QIU-PEI TAN ${ }^{2 *}$, WEI-GUO SUI ${ }^{1}$, WEN-BIAO CHEN ${ }^{3}$, \\ WU-JIAN PENG ${ }^{3}$, XING-CHAO LIU ${ }^{2}$ and YONG DAI ${ }^{3}$ \\ ${ }^{1}$ Central Laboratory of Guilin 181st Hospital, Key laboratory of Metabolic Diseases Research; \\ ${ }^{2}$ Clinical Laboratory of 181st Hospital, Guilin, Guangxi 541002; ${ }^{3}$ The Second Clinical Medical College \\ of Jinan University, Shenzhen People's Hospital, Shenzhen, Guangdong 518020, P.R. China
}

Received August 16, 2016; Accepted November 30, 2016

DOI: 10.3892/br.2016.835

\begin{abstract}
The aim of the present study was to examine differentially expressed proteome profiles for candidate biomarkers in peripheral blood mononuclear cells (PBMCs) of liver failure (LF) patients. Ten patients were diagnosed as LF and 10 age- and gender-matched subjects were recruited as healthy controls. Isobaric tags for relative and absolute quantitation (iTRAQ)-based quantitative proteomic technology is efficiently applicable for identification and relative quantitation of the proteomes of PBMCs. Eight-plex iTRAQ coupled with strong cation exchange chromatography, and liquid chromatography coupled with tandem mass spectrometry were used to analyze total proteins in LF patients and healthy control subjects. Molecular variations were detected using the iTRAQ method, and western blotting was used to verify the results. LF is a complex type of medical emergency that evolves following a catastrophic insult to the liver, and its outcome remains the most ominous of all gastroenterologic diseases. Serious complications tend to occur during the course of the disease and further exacerbate the problems. Using the iTRAQ method, differentially expressed proteome profiles of LF patients were determined. In the present study, 627 proteins with different expression levels were identified in LF patients compared with the control subjects; with 409 proteins upregulated and 218 proteins downregulated. Among them, four proteins were significantly differentially
\end{abstract}

Correspondence to: Dr Yong Dai, The Second Clinical Medical College of Jinan University, Shenzhen People's Hospital, 1017 Dongmen Beilu, Luohu, Shenzhen, Guangdong 518020, P.R. China

E-mail: daiyong22@aliyun.com

*Contributed equally

Key words: isobaric tags for relative and absolute quantitation, proteomics, tandem mass spectrometry, liver failure, biomarker expressed; acylaminoacyl-peptide hydrolase and WW domain binding protein 2 were upregulated, and resistin and tubulin $\beta$ $2 \mathrm{~A}$ class IIa were downregulated. These proteins demonstrated differences in their expression levels compared with other proteins with normal expression levels and the significant positive correlation with LF. The western blot results were consistent with the results from iTRAQ. Thus, investigation of the molecular mechanism of the proteins involved in LF may facilitate an improved understanding of the pathogenesis of LF and elucidation of novel biomarker candidates.

\section{Introduction}

Liver failure (LF) is a complex medical emergency that evolves following a catastrophic insult to the liver with an outcome that remains the most ominous of all the gastroenterologic diseases. LF is severe liver damage resulting from various factors, which cause obstruction or decompensation of function, such as composition, detoxifucation, drainage and biotransformation. Various clinical syndromes appear, including the obstruction of coagulation mechanisms, icterus, hepatic encephalopathy and ascites. Serious complications, such as hepatic encephalopathy and renal inadequacy tend to occur during the course of the disease and further exacerbate clinical syndromes. It is conventionally defined by an arterial oxygen tension $\left(\mathrm{P}_{\mathrm{a}} \mathrm{O}_{2}\right)<8.0 \mathrm{kPa}(60 \mathrm{mmHg})$ and/or an arterial carbon dioxide tension $\left(\mathrm{P}_{\mathrm{a}} \mathrm{CO}_{2}\right)>6.0 \mathrm{kPa}(45 \mathrm{mmHg})$, and a serum bilirubin level $\geq 12.0 \mathrm{mg} / \mathrm{dl}$. The prognosis of patients with severe liver injury, particularly with LF, largely depends upon the regenerative capacity of hepatocytes during comprehensive treatment. Liver transplantation improves survival and quality of life. However, treatment is futile in certain patients, but it is difficult to identify these patients a priori. In clinical practice, serum $\alpha$-fetoprotein is often used as a predictive biomarker for monitoring the prognosis of patients with LF, as it reflects the regeneration of hepatocytes in response to liver injury (1-3). Although the most important function of telomerase is associated with cell proliferation and regeneration, to the best of our knowledge, there are no studies regarding the association of the prognosis of LF and telomerase 
activation. Thus, identification of molecular markers is required and novel treatments against this disease must be developed. In addition, improved non-invasive methods of detecting LF are urgently required in order to influence the survival of the increasing numbers of individuals affected by this disease.

The precise molecular pathogenic mechanism of LF remains unknown. Development of biomarkers for a deeper understanding of LF pathogenesis and improving diagnosis, prognosis, and treatment remains one of the main goals and challenges in LF research. Biomarkers within the blood and urine reflect the status and possible future progression of a disease (4). Aberrant functions of the lymphocytic regulatory pathway are extensively involved in the pathological mechanism of certain diseases (5); therefore, peripheral blood mononuclear cells (PBMCs) are an attractive sample source in such studies. Proteomic analysis is a research method that catalogs all of the proteins within cells and organisms. Recent advancements in quantitative and large-scale proteomic methods may be used to optimize the clinical application of biomarkers (6). Furthermore, advancements of proteomic techniques contribute to the identification of clinically useful biomarkers and clarify the molecular mechanisms of disease pathogenesis using body fluids, such as serum, as well as tissue samples and cultured cells.

Proteomics analysis is a powerful technology used in a myriad of studies, including those focused on liver diseases (7-11). The isobaric tags for relative and absolute quantitation (iTRAQ) method allows a more comprehensive analysis. This method has a high sensitivity and it is possible to detect low-abundance proteins. iTRAQ has increasingly been applied in biomarker research in various sample sources for various disease states (12-14). Charlton et al (15) compared the protein expression profiles in four groups of liver tissue samples from obese patients using the combination of iTRAQ with liquid chromatography (LC)-mass spectrometry (MS)/MS. The authors identified a total of 1,362 hepatic-expressed proteins, and identified two important proteins. Niu et al performed various in vitro proteomic investigations of Hepatitis B virus (HBV)-infected HepG2 hepatoma cells to evaluate the protein changes associated with the virus infection. Using the combined methods of iTR AQ with 2D-LC-MS/MS, the authors compared the protein expression in non-infected HepG2 with that in HBV-infected HepG2 cells to identify various proteins that were downregulated in the HBV-infected cells, including S100 calcium-binding protein A6 and Annexin A2 $(16,17)$.

In the present study, iTRAQ technology was used to analyze the total proteins in PBMCs of LF patients. The aim was to identify the differences in PBMC protein levels that were closely associated with the progression of LF. Further investigation into the molecular mechanism of the proteins involved may improve understanding of the pathogenesis of LF and facilitate development of novel approaches to diagnose and treat LF.

\section{Materials and methods}

Main reagents. Triton X-100 was purchased from GE Healthcare (Waukesha, WI, USA). Triethylammonium bicarbonate buffer was acquired from Sigma-Aldrich (Merck Millipore, Darmstadt, Germany). ZipTip Pipette Tips and
Milli-Q water were obtained from EMD Millipore (Billerica, MA, USA). The iTRAQ Reagent-8 Plex Multiplex kit was acquired from Applied Biosystems (Thermo Fisher Scientific, Inc., Waltham, MA, USA) and Strata-X 33 Polymeric Reversed Phase was purchased from Phenomenex (Los Angeles, CA, USA). All other reagents were acquired from commercial sources.

Patients and healthy controls. Ten patients (6 male and 4 female; aged 23-57 years) were diagnosed as LF between January and December 2014, and 10 age- and gender-matched subjects were recruited as healthy controls. HBV-associated LF refers to patients with LF caused by chronic HBV infection. The 10 patients and 10 healthy control subjects were from Shenzhen People's Hospital (Shenzhen, China). The diagnosis of LF was confirmed by pathologic diagnosis and clinical evidence.

The control subjects were recruited and a general health checkup program confirmed that there was no clinical evidence of LF. All participants were informed of their participation rights and written informed consent was obtained. The present study was performed in accordance with the Helsinki Declaration and approved by the Regional Ethics Committee.

PBMC isolation, protein extraction and quantitation. One $10-\mathrm{ml}$ fasting venous blood sample was collected in heparinized vacutainers from each enrolled subject. PBMCs were isolated with lymphocyte-H medium (Cedarlane Labs, Hornby, ON, Canada) according to the manufacturer's instruction. The total protein of PBMCs was extracted, and their concentration was measured using a BCA protein assay kit (Pierce; Thermo Fisher Scientific, Inc.) according to the manufacturer's instruction. The proteins in the supernatant were maintained at $-80^{\circ} \mathrm{C}$ for further analysis.

iTRAQ labeling and strong cation exchange (SCX) chromatography fractionation. Total protein $(100 \mu \mathrm{g})$ from the PBMCs of the 10 LF patients and 10 healthy control subjects was digested separately with Trypsin Gold (Promega Corporation, Madison, WI, USA) with the ratio of protein:trypsin 30:1 at $37^{\circ} \mathrm{C}$ for $16 \mathrm{~h}$. Following trypsin digestion, peptides were dried by vacuum centrifugation at $2,000 \mathrm{x} g$ at room temperature for $10 \mathrm{~min}$. Peptides were reconstituted in $0.5 \mathrm{M}$ triethyl-ammonium bicarbonate buffer and processed according to the manufacturer's protocol for the 8-plex iTRAQ reagent. Briefly, one unit of iTRAQ reagent was thawed and reconstituted in $24 \mu 1$ isopropanol. Samples were labeled with the iTRAQ tags as follows: Sample 113 and sample 115. The peptides were labeled with the isobaric tags and incubated at room temperature for $2 \mathrm{~h}$. The labeled peptide mixtures were then pooled and dried by vacuum centrifugation at $2,000 \mathrm{x} \mathrm{g}$ at room temperature for $10 \mathrm{~min}$.

SCX chromatography was performed with a LC-20AB high-performance LC (HPLC) Pump system (Shimadzu Corp., Kyoto, Japan). The iTR AQ-labeled peptide mixtures were reconstituted with $4 \mathrm{ml}$ buffer A $\left(25 \mathrm{mM} \mathrm{NaH}_{2} \mathrm{PO}_{4}\right.$ in $25 \% \mathrm{ACN}, \mathrm{pH} 2.7)$ and loaded onto a $4.6 \times 250 \mathrm{~mm}$ Ultremex SCX column containing 5- $\mu \mathrm{m}$ particles (Phenomenex). The peptides were eluted at a flow rate of $1 \mathrm{ml} / \mathrm{min}$ with a gradient of buffer A for $10 \mathrm{~min}, 5-60 \%$ buffer B $\left(25 \mathrm{mM} \mathrm{NaH}_{2} \mathrm{PO}_{4}, 1 \mathrm{M}\right.$ 
$\mathrm{KCl}$ in $25 \% \mathrm{ACN}, \mathrm{pH} 2.7$ ) for $27 \mathrm{~min}$ and $60-100 \%$ buffer $\mathrm{B}$ for 1 min. The system was then maintained at $100 \%$ buffer B for $1 \mathrm{~min}$ before equilibrating with buffer $\mathrm{A}$ for $10 \mathrm{~min}$ prior to the next injection. Elution was monitored by measuring the absorbance at $214 \mathrm{~nm}$ with ultraviolet-visible spectrophotometry, and fractions were collected at 1-min intervals. The eluted peptides were pooled into 20 fractions, desalted with a Strata X C18 column (Phenomenex) and vacuum-dried.

$L C$-MS/MS analysis based on Q-EXACTIVE. Each fraction was resuspended in buffer A ( $2 \% \mathrm{ACN}, 0.1 \% \mathrm{FA})$ and centrifuged at 20,000 x g for $10 \mathrm{~min}$ at $4^{\circ} \mathrm{C}$, the final concentration of peptide was $\sim 0.5 \mu \mathrm{g} / \mu \mathrm{l}$ on average. Supernatant $(10 \mu \mathrm{l})$ was loaded onto an LC-20AD nano HPLC (Shimadzu Corp., Kyoto, Japan) by the autosampler onto a 2-cm C18 trap column (inner diameter, $200 \mu \mathrm{m}$ ). Then, the peptides were eluted onto a 10-cm analytical C18 column (inner diameter, $75 \mu \mathrm{m}$ ) packed in-house. The samples were loaded at $8 \mu \mathrm{l} / \mathrm{min}$ for $4 \mathrm{~min}$, then the 44-min gradient was run at $300 \mathrm{nl} / \mathrm{min}$ starting from 2 to $35 \%$ solvent B (98\% ACN, $0.1 \%$ fatty acid), followed by a 2 -min linear gradient to $80 \%$ and maintenance at $80 \%$ solvent B for $4 \mathrm{~min}$, and finally returning to $5 \%$ in $1 \mathrm{~min}$.

The peptides were subjected to nanoelectrospray ionization followed by MS/MS in a Q-EXACTIVE (Thermo Fisher Scientific, Inc.) coupled online to the HPLC. Intact peptides were detected in the orbitrap (Thermo Fisher Scientific, Inc.) at a resolution of 70,000. Peptides were selected for MS/MS using high-energy collision dissociation operating mode with a normalized collision energy setting of 27.0; ion fragments were detected in the orbitrap at a resolution of 17,500. A data-dependent procedure that alternated between one MS scan followed by $15 \mathrm{MS} / \mathrm{MS}$ scans was applied for the 15 most abundant precursor ions above a threshold ion count of 20,000 in the MS survey scan with a subsequent dynamic exclusion duration of $15 \mathrm{sec}$. The electrospray voltage applied was $1.6 \mathrm{kV}$. Automatic gain control (AGC) was used to optimize the spectra generated by the orbitrap. The AGC target for full MS was $3 \mathrm{e} 6$ and 1e5 for MS2. For the MS scans, the mass to charge ratio $(\mathrm{m} / \mathrm{z})$ scan range was $350-2,000 \mathrm{Da}$, and for the MS2 scans, the $\mathrm{m} / \mathrm{z}$ scan range was 100-1,800.

Western blot analysis. The protein abundance of the pooled samples previously analyzed by iTRAQ LC-MS/MS was confirmed essentially as previously described using western blotting (18). Four protein samples $(35 \mu \mathrm{g})$ were added into electrophoretic buffer containing $\beta$-mercaptoethanol prior to SDS-PAGE. GAPDH (Kangcheng, Shanghai, China) served as a loading control. The primary antibody (dilution, 1:250; cat. no. ab89835) for ADP-ribosylation factor 1 was obtained from Abcam (Cambidge, MA, USA) and the peroxidase-conjugated goat anti-rabbit IgG secondary antibody (dilution, 1:10,000) was obtained from SouthernBiotech (Birmingham, AL, USA; cat. no. A21020). The band intensity of western blot analysis was repeated three times using ImageJ software (National Institutes of Health, Bethesda, MD, USA).

Statistical analysis. Protein identification was performed using Mascot version 2.3.02 (Matrix Science, London, UK). Peptide sequences were searched against the non-redundant National Center for Biotechnology Information database. For protein quantitation, it was required that a protein contained at least two unique peptides. The quantitative protein ratios were weighted and normalized by the median ratio in Mascot. Only ratios with $\mathrm{P}<0.05$ and fold-change $>1.2$ were considered to be statistically significant. Search criteria were set to permit a maximum of 1 missed cleavage. The following peptide modifications were also allowed: Gln $\rightarrow$ pyro-Glu, iTRAQ 8plex, Phospho. Automatic isotope correction was performed using the two software packages using the values supplied with the Applied Biosystems reagents. The PANTHER database (http://www.pantherdb.org/panther/) was then used to establish the molecular function, biological process and signaling pathway associated with each individual protein. GO and KEGG pathway mapping were performed by web-accessible DAVID annotation system (version 6.7; https://david-d.ncifcrf. gov/).

\section{Results}

Protein expression profile. Compared with the control group, a total of 627 differently expressed proteins were detected, of which 409 proteins showed increased expression levels and 218 proteins showed decreased expression levels in LF (Table I). The protein ratio distribution of these proteins is illustrated in Fig. 1. Relative quantification of proteins was based on the ratio of peak areas from the MS/MS spectra, and the $\mathrm{m} / \mathrm{z}$ of LF patients and control subjects were involved in the present study.

Gene Ontology (GO) and Kyoto Encyclopedia of Genes and Genomes (KEGG) pathway analysis. The functions of the differently expressed proteins were analyzed using the GO and KEGG pathways annotation system. The proteins produced a total of $420 \mathrm{GO}$ terms in the LF group (Table II), including 284 in biological process, 74 in cellular component and 62 in molecular function. The results indicate that a set of highly abundant and significantly differentially expressed proteins may promote the progression of LF patients. In addition, 14 KEGG pathways of the differently expressed proteins in LF were obtained (Table III).

Western blot analysis. Four proteins, acylaminoacyl-peptide hydrolase (APEH; 1.309), WW domain binding protein 2 (WBP2; 1.385), resistin $(0.747)$ and tubulin $\beta 2 \mathrm{~A}$ class IIa (TUBB2A; 0.764), were selected from the 627 proteins with variable expression levels. These differently expressed proteins between the LF and control groups were verified by western blotting (Table IV and Fig. 2), which indicated a similar relative expression level when compared with the iTRAQ LC-MS/MS analysis.

\section{Discussion}

Protein quantification has become an important and, in many cases, critical component of modern MS-based proteomic research $(19,20)$. Proteomics is the term used for exhaustive analysis of protein structure and function. It is useful for elucidation of the pathology and identification of disease markers for liver diseases. PBMCs are often used as clinical samples, rather than tissue, as less invasive methods may be used to 
Table I. LF proteome.

A, Top 30 increased proteins in LF

No.

1 splP22392INDKB_HUMAN

2 splQ9UM07IPADI4_HUMAN

3 spIQ9CQC6IBZW1_MOUSE

4 splQ96DA6ITIM14_HUMAN

5 splP52790IHXK3_HUMAN

6 splP80188INGAL_HUMAN

7 splP25774ICATS_HUMAN

8 splP12429|ANXA3_HUMAN

9 splP37837ITALDO_HUMAN

10 splP41218IMNDA_HUMAN

11 splQ9ULZ3IASC_HUMAN

12 splQ4R6V2ITCPE_MACFA

13 splP26583|HMGB2_HUMAN

14 splQ9UBW5IBIN2_HUMAN

15 splQ6P4A8IPLBL1_HUMAN

16 splA6NI72INCF1B_HUMAN

17 splP39687IAN32A_HUMAN

18 splQ92688IAN32B_HUMAN

19 splP61586IRHOA_HUMAN

20 splP20700ILMNB1_HUMAN

21 splO75962ITRIO_HUMAN

22 splQ92905ICSN5_HUMAN

23 spIP18433|PTPRA_HUMAN

24 splQ8BL97ISRSF7_MOUSE

25 splP48595ISPB10_HUMAN

26 splP50402|EMD_HUMAN

27 splO96006IZBED1_HUMAN

28 splP09668|CATH_HUMAN

29 splP50395|GDIB_HUMAN

30 splO73777|IF4G2_CHICK
Description

Nucleoside diphosphate kinase B

Protein-arginine deiminase type- 4

Basic leucine zipper and $\mathrm{W} 2$ domain-containing protein 1

Mitochondrial import inner membrane translocase subunit TIM14

Hexokinase-3

Neutrophil gelatinase-associated lipocalin

Cathepsin S

Annexin A3

Transaldolase

Myeloid cell nuclear differentiation antigen

Apoptosis-associated speck-like protein containing a CARD

T-complex protein 1 subunit epsilon

High mobility group protein B2

Bridging integrator 2

Phospholipase B-like 1

Putative neutrophil cytosol factor 1B

Acidic leucine-rich nuclear phosphoprotein 32 family member A

Acidic leucine-rich nuclear phosphoprotein 32 family member B

Transforming protein RhoA

$$
\text { Lamin-B1 }
$$

Triple functional domain protein

COP9 signalosome complex subunit 5

Receptor-type tyrosine-protein phosphatase $\alpha$

Serine/arginine-rich splicing factor 7

Serpin B10

Emerin

Zinc finger BED domain-containing protein 1

Pro-cathepsin $\mathrm{H}$

Rab GDP dissociation inhibitor $\beta$

Eukaryotic translation initiation factor $4 \gamma 2$ (Fragment)
Ratio of LF

to control

3.953

3.509

3.431

3.297

2.957

2.928

2.8

2.631

2.595

2.55

2.514

2.362

2.361

2.355

2.339

2.338

2.295

2.295

2.292

2.249

2.233

2.201

2.187

2.186

2.171

2.17

2.162

2.146

2.091

B, Top 30 decreased proteins in LF

Ratio of LF

No.

Accession

Description

to control

\begin{tabular}{lc}
\hline 1 & splQ9Y2R4IDDX52_HUMAN \\
2 & splO75015|FCG3B_HUMAN \\
3 & splP12236IADT3_HUMAN \\
4 & splQ9NTG7ISIR3_HUMAN \\
5 & splP14222|PERF_HUMAN \\
6 & splP20718|GRAH_HUMAN \\
7 & splP04264|K2C1_HUMAN \\
8 & splP19086|GNAZ_HUMAN \\
9 & splP11166|GTR1_HUMAN \\
10 & splQ969X1|LFG3_HUMAN \\
11 & splQ15050|RRS1_HUMAN \\
12 & splP12544|GRAA_HUMAN \\
13 & splP14209|CD99_HUMAN
\end{tabular}

Probable ATP-dependent RNA helicase DDX52

0.078

Low affinity immunoglobulin gamma Fc region receptor III-B

0.211

ADP/ATP translocase 3

0.277

NAD-dependent protein deacetylase sirtuin-3, mitochondrial

0.298

Perforin-1

0.346

Granzyme H

0.39

Keratin, type II cytoskeletal 1

0.419

Guanine nucleotide-binding protein G(z) subunit $\alpha$

0.433

Solute carrier family 2, facilitated glucose transporter member $1 \quad 0.434$

Protein lifeguard 3

0.437

Ribosome biogenesis regulatory protein homolog

0.442

Granzyme A

0.448

CD99 antigen

0.45 
Table I. Continued

B, Top 30 decreased proteins in LF

\begin{tabular}{|c|c|c|c|}
\hline No. & Accession & Description & $\begin{array}{c}\text { Ratio of LF } \\
\text { to control }\end{array}$ \\
\hline 14 & splP07996ITSP1_HUMAN & Thrombospondin-1 & 0.454 \\
\hline 15 & splP05106IITB3_HUMAN & Integrin $\beta-3$ & 0.454 \\
\hline 16 & splP02788ITRFL_HUMAN & Lactotransferrin & 0.479 \\
\hline 17 & splP35527IK1C9_HUMAN & Keratin, type I cytoskeletal 9 & 0.487 \\
\hline 18 & splO15533ITPSN_HUMAN & Tapasin & 0.491 \\
\hline 19 & splO60704|TPST2_HUMAN & Protein-tyrosine sulfotransferase 2 & 0.504 \\
\hline 20 & splQ9BVC6ITM109_HUMAN & Transmembrane protein 109 & 0.506 \\
\hline 21 & splP37840|SYUA_HUMAN & $\alpha$-synuclein & 0.511 \\
\hline 22 & splQ08AF3ISLFN5_HUMAN & Schlafen family member 5 & 0.514 \\
\hline 23 & splP16109|LYAM3_HUMAN & P-selectin & 0.517 \\
\hline 24 & splP01871|IGHM_HUMAN & $\operatorname{Ig} \mu$ chain $\mathrm{C}$ region & 0.523 \\
\hline 25 & splP50336IPPOX_HUMAN & Protoporphyrinogen oxidase & 0.529 \\
\hline 26 & splP02788ITRFL_HUMAN & Lactotransferrin & 0.543 \\
\hline 27 & sp|P68872|HBB_PANPA & Hemoglobin subunit $\beta$ & 0.548 \\
\hline 28 & splQ9Y6W5IWASF2_HUMAN & Wiskott-Aldrich syndrome protein family member 2 & 0.549 \\
\hline 29 & splP18428ILBP_HUMAN & Lipopolysaccharide-binding protein & 0.556 \\
\hline 30 & splQ99798IACON_HUMAN & Aconitate hydratase, mitochondrial & 0.556 \\
\hline
\end{tabular}

Ratios with $\mathrm{P}<0.05$ and fold-change $>1.2$ were considered to be statistically significant. The top 30 proteins that were increased or decreased in LF according to iTRAQ were extracted. LF, liver failure; iTRAQ, isobaric tags for relative and absolute quantitation.

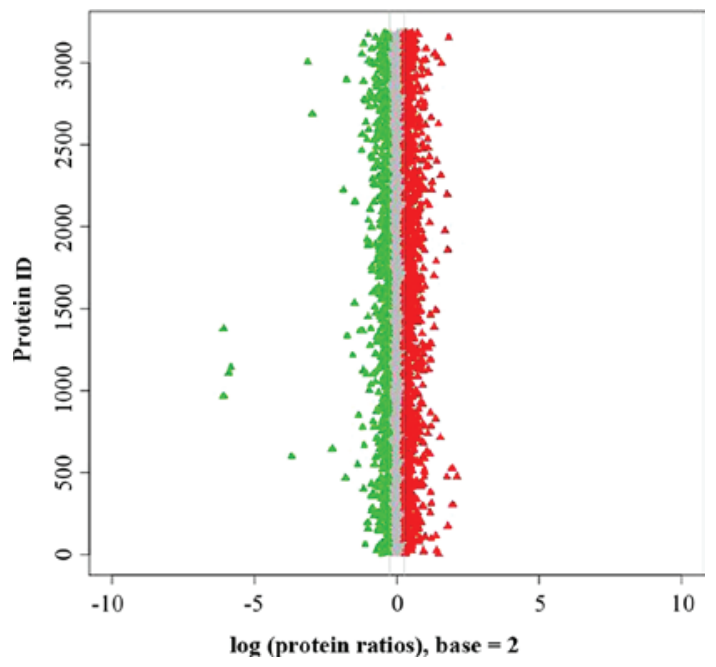

Figure 1. Protein ratio distribution of differentially expressed proteins in liver failure. Green, downregulated differently expressed proteins; red, upregulated differently expressed proteins.

obtain them. If a biomarker associated with the pathology, disease progression or efficacy of treatment is identified in PBMCs, it may be easily applied for early or differential diagnosis of diseases. Of these, iTRAQ, which enables the parallel comparison of protein abundance by measuring the peak intensities of reporter ions released from iTRAQ-tagged peptides, has the potential to be a key tool in the area of quantitative proteomic study. In the current study, iTR AQ technology was adopted to quantitatively analyze the proteomics of PBMCs from LF patients and healthy control subjects. As a result, 627 proteins involving different biological functions and cellular locations were identified. Among these proteins, four proteins were significantly differentially expressed; APEH and WBP2 were upregulated, and resistin and TUBB2A were downregulated. It provided additional proof that the iTRAQ technique accurately quantifies relative changes in protein abundance of PBMCs, which has been demonstrated to be useful in detecting pathological stages or prognosis in certain diseases, such as osteoarthritis $(21,22)$.

Resistin is cysteine-rich protein belonging to the RELM family. The genetic structure of the Retn gene varies between mammals and the similarity in the coding sequence ranges from $\sim 60 \%$ for rodents to $80 \%$ for livestock (23). The expression of this gene in rodents occurs predominantly in mature adipocytes, although it was also identified in other tissues. Retn is considered to be a factor linking obesity and insulin resistance. In obesity, its expression increases, leading to enhanced resistance of tissues to insulin (24). TUBB2A is thought to comprise $\sim 30 \%$ of all $\beta$-tubulin within the brain (25) and contributes to the growing list of tubulin gene mutations that are associated with impaired brain development in humans. Increased expression levels of TUBB2A have been correlated with decreased drug sensitivity in paclitaxel-resistant cell lines (26). A previous study demonstrated the potential of a model for gene perturbation studies by demonstrating that decreased expression levels of TUBB2A result in significantly increased sensitivity of neurons to paclitaxel (27). 
Table II. Up- and downregulated protein annotation terms of the GO molecular function, cellular component and biological process categories in LF.

\begin{tabular}{|c|c|c|c|}
\hline Term & P-value & Term & P-value \\
\hline \multicolumn{4}{|l|}{ Biological process } \\
\hline Transport & $6.41 \mathrm{E}-05$ & Multi-organism process & 0.001086326 \\
\hline Establishment of localization & $6.58 \mathrm{E}-05$ & $\begin{array}{l}\text { DNA damage response, signal transduction } \\
\text { by p53 class mediator }\end{array}$ & 0.001163164 \\
\hline Immune system process & 0.000134159 & Vesicle-mediated transport & 0.001412972 \\
\hline Immune response & 0.000164528 & Intracellular transport & 0.001540664 \\
\hline Establishment of localization in cell & 0.000176366 & Release of sequestered calcium ion into cytosol & 0.001593621 \\
\hline Negative regulation of molecular function & 0.000205286 & Regulation of sequestering of calcium ion & 0.001593621 \\
\hline Negative regulation of catalytic activity & 0.000304827 & Negative regulation of sequestering of calcium ion & 0.001593621 \\
\hline Localization & 0.000494539 & Cytosolic calcium ion transport & 0.001593621 \\
\hline Vesicle fusion & 0.00085815 & Calcium ion transport into cytosol & 0.001593621 \\
\hline Cellular localization & 0.000951991 & Signal transduction by p53 class mediator & 0.001850301 \\
\hline \multicolumn{4}{|l|}{ Molecular function } \\
\hline Cation transmembrane transporter activity & 0.000354884 & endopeptidase regulator activity & 0.006225752 \\
\hline $\begin{array}{l}\text { Hydrogen ion transmembrane } \\
\text { transporter activity }\end{array}$ & 0.001472303 & Immunoglobulin binding & 0.006472871 \\
\hline $\begin{array}{l}\text { Inorganic cation transmembrane } \\
\text { transporter activity }\end{array}$ & 0.002536343 & $\begin{array}{l}\text { Substrate-specific transmembrane } \\
\text { transporter activity }\end{array}$ & 0.006837942 \\
\hline Peptidase regulator activity & 0.002777008 & Endopeptidase inhibitor activity & 0.007710339 \\
\hline Transporter activity & 0.003623169 & Peptidase inhibitor activity & 0.007710339 \\
\hline Peptide transporter activity & 0.004093554 & Monosaccharide binding & 0.008311587 \\
\hline Amide transmembrane transporter activity & 0.004093554 & Ubiquinol-cytochrome-c reductase activity & 0.008525874 \\
\hline Enzyme inhibitor activity & 0.004329426 & Ferric iron binding & 0.008525874 \\
\hline Substrate-specific transporter activity & 0.004897002 & $\begin{array}{l}\text { Oxidoreductase activity, acting on } \\
\text { diphenols and related substances as donors }\end{array}$ & 0.008525874 \\
\hline Ion transmembrane transporter activity & 0.005584917 & $\begin{array}{l}\text { Oxidoreductase activity, acting on diphenols and } \\
\text { related substances as donors, cytochrome as acceptor }\end{array}$ & 0.008525874 \\
\hline \multicolumn{4}{|l|}{ Cellular component } \\
\hline Cytoplasmic vesicle & 0.000318 & Phagocytic vesicle & 0.003696 \\
\hline Vesicle & 0.000409 & endosomal part & 0.005078 \\
\hline Cytoplasmic membrane-bounded vesicle & 0.000483 & Proteasome complex & 0.005818 \\
\hline Secretory granule & 0.000497 & Endosome & 0.006978 \\
\hline Cytoplasmic vesicle part & 0.000632 & Proteasome core complex, alpha-subunit complex & 0.008175 \\
\hline Membrane-bounded vesicle & 0.000705 & TAP complex & 0.00878 \\
\hline Platelet alpha granule & 0.00168 & Cytoplasmic vesicle membrane & 0.009488 \\
\hline Secondary lysosome & 0.002441 & Endosome membrane & 0.010481 \\
\hline Phagolysosome & 0.002441 & Vesicle membrane & 0.011522 \\
\hline Endocytic vesicle & 0.002743 & Organelle envelope lumen & 0.011583 \\
\hline
\end{tabular}

$\mathrm{P}<0.05$ was considered to be statistically significant. The top $20 \mathrm{GO}$ terms were listed in LF according to molecular function, cellular component and biological process. GO, Gene Ontology; LF, liver failure.

The role of WBP-2 is as a coactivator for estrogen receptor and progesterone receptor transactivation pathways (28). APEH has been postulated to serve as a key regulator of N-terminal acetylated proteins (29). As $>80 \%$ of proteins in human cells are $\mathrm{N}$-terminal acetylated $(30,31)$ and protein acetylation is implicated in a variety of essential cellular pathways (32), it is feasible that APEH is involved in these processes. APEH, one of the four members of the prolyl oligopeptidase class, catalyses the removal of $\mathrm{N}$-acetylated amino acids from acetylated peptides and it has been postulated to be key in protein degradation machinery. Disruption of protein turnover has been established as an effective strategy to downregulate the ubiquitin proteasome system and as a promising approach in anticancer therapy. APEH may be an upstream modulator of the proteasome (33).

To establish the biological roles of the proteins from LF, GO enrichment and KEGG pathway analyses were performed. GO 
Table III. The Kyoto Encyclopedia of Genes and Genomes pathways of the differently expressed proteins in liver failure.

\begin{tabular}{lll}
\hline Pathway & \multicolumn{1}{c}{ P-value } & Pathway ID \\
\hline Spliceosome & 0.0004265046 & ko03040 \\
Cardiac muscle contraction & 0.0006583187 & ko04260 \\
Proteasome & 0.002674917 & ko03050 \\
Oxidative phosphorylation & 0.004688983 & ko00190 \\
Parkinson's disease & 0.0061224 & ko05012 \\
Valine, leucine and & 0.008369825 & ko00290 \\
isoleucine biosynthesis & & \\
Malaria & 0.01015188 & ko05144 \\
Pathogenic Escherichia coli & 0.0161563 & ko05130 \\
infection & & \\
Leukocyte transendothelial & 0.01934782 & ko04670 \\
migration & & \\
Alzheimer's disease & 0.01948298 & ko05010 \\
Glycolysis/gluconeogenesis & 0.02570021 & ko00010 \\
Osteoclast differentiation & 0.02836594 & ko04380 \\
Phagosome & 0.02839378 & ko04145 \\
Galactose metabolism & 0.0475115 & ko00052 \\
\hline
\end{tabular}

$\mathrm{P}<0.05$ was considered to indicate a statistically significant difference.

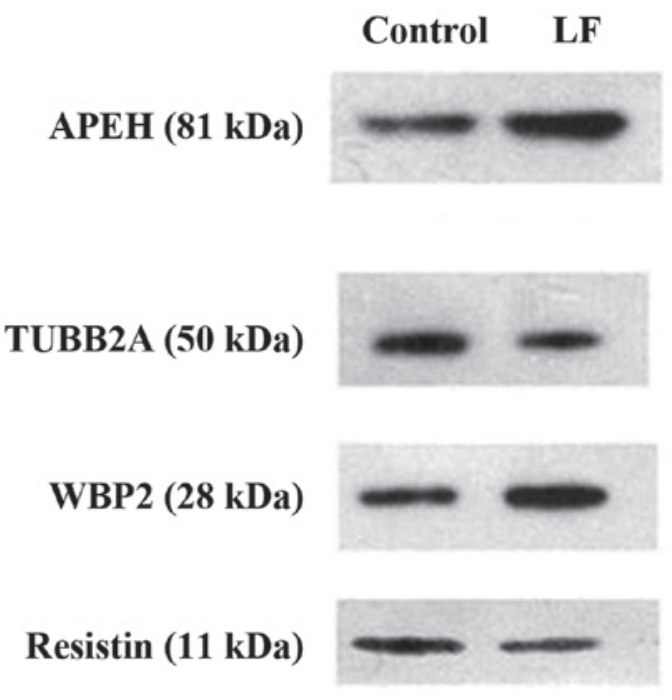

Figure 2. Western blot analysis of APEH, WBP2, Resistin and TUBB2A in the LF and control groups. APEH, acylaminoacyl-peptide hydrolase; WBP2, WW domain binding protein 2; TUBB2A, tubulin $\beta$ 2A class IIa; LF, liver failure.

categories were separated into three groups: Molecular function, biological process and cellular component. The present study identified GO terms for molecular function significantly enriched in protein binding (GO:0005515; $\mathrm{P}=0.02145$ ) involving $T U B B 2 A$ and WBP2, and for cellular component, the enriched GO terms were cytoplasm (GO:0005737; $\mathrm{P}=0.02634)$ involving APEH and TUBB2A. KEGG analysis was performed with $\mathrm{P}<0.05$ as the criteria for significant pathway identification. The significant pathway in the KEGG analysis was identified as phagosome $(\mathrm{P}=0.02839)$ involving TUBB2A.
Table IV. Western blot analysis of APEH, WBP2, resistin and TUBB2A in the LF and control groups.

\begin{tabular}{lcc}
\hline Name & $\begin{array}{c}\text { Control } \\
\text { (relative expression) }\end{array}$ & $\begin{array}{c}\text { LF } \\
\text { (relative expression) }\end{array}$ \\
\hline Resistin & 1.000 & 0.715 \\
APEH & 1.000 & 1.297 \\
TUBB2A & 1.000 & 0.738 \\
WBP2 & 1.000 & 1.072
\end{tabular}

APEH, acylaminoacyl-peptide hydrolase; WBP2, WW domain binding protein 2 ; TUBB2A, tubulin $\beta$ 2A class IIa; LF, liver failure.

Phagocytosis is the process of a cell taking in relatively large particles, and is a central mechanism in tissue remodeling, inflammation and defense against infectious agents. A phagosome is formed when the specific receptors on the phagocyte surface recognize ligands on the particle surface. Following formation, nascent phagosomes progressively acquire digestive characteristics.

In conclusion, proteomic technologies based on MS have been developed, and the reliability of these technologies continues to improve. Such advancements in proteomic techniques may contribute to the identification of clinically useful biomarkers and the elucidation of the molecular mechanisms involved in disease pathogenesis. Therefore, a more sensitive detection system to search for biomarkers is required, and this may allow clinically useful markers for all liver diseases to be identified. Proteins are assumed to be key molecules that define the characteristics and dynamics of cells, and control biological reactions. Therefore, investigation of changes in protein expression levels is particularly important in understanding disease pathology. A limitation of the current study is that it did not discuss each of the candidate proteins in detail. The aim of this preliminary study was to focus on delineating primary comparative protein profiles of LF patients and healthy control subjects using iTRAQ technology. In future, a large-scale clinical study is required to investigate useful biomarkers of LF. This may result in a novel method for diagnosing LF. In addition, the current study demonstrates the potential application of iTRAQ-based quantitative proteomics for identifying protein changes and detecting notable biomarker candidates in certain diseases. Thus, identification and evaluation of an easily measurable biomarker is imperative. A combination of conventional markers with newly identified markers, the variation of which was confirmed in the present study, may improve diagnosis of the LF disease state and the capacity for prognosis.

\section{Acknowledgements}

The authors would like to thank the patients with LF and the healthy volunteers who participated in the present study. The study was performed by Beijing Genomics Institute and supported by the Guangxi Key Laboratory Construction Project Plan (grant no. 15-140-10) and the Clinical Research Program of Guilin 181st Hospital (Guilin, China; grant no. [2008]125). 


\section{References}

1. Schiødt FV, Ostapowicz G, Murray N, Satyanarana R, Zaman A, Munoz S and Lee WM: Alpha-fetoprotein and prognosis in acute liver failure. Liver Transpl 12: 1776-1781, 2006.

2. Li Q, Yuan GY, Tang KC, Liu GW, Wang R and Cao WK: Prognostic factors for chronic severe hepatitis and construction of a prognostic model. Hepatobiliary Pancreat Dis Int 7: 40-44, 2008.

3. Du WB, Pan XP and Li LJ: Prognostic models for acute liver failure. Hepatobiliary Pancreat Dis Int 9: 122-128, 2010.

4. Henriksen K, O'Bryant SE, Hampel H, Trojanowski JQ, Montine TJ, Jeromin A, Blennow K, Lönneborg A, Wyss-Coray T, Soares H, et al; Blood-Based Biomarker Interest Group: The future of blood-based biomarkers for Alzheimer's disease. Alzheimers Dement 10: 115-131, 2014.

5. Chang X, Cui Y, Zong M, Zhao Y, Yan X, Chen Y and Han J: Identification of proteins with increased expression in rheumatoid arthritis synovial tissues. J Rheumatol 36: 872-880, 2009.

6. Zhou L, Beuerman RW, Chan CM, Zhao SZ, Li XR, Yang H, Tong L, Liu S, Stern ME and Tan D: Identification of tear fluid biomarkers in dry eye syndrome using iTRAQ quantitative proteomics. J Proteome Res 8: 4889-4905, 2009.

7. Ren F, Chen Y, Wang Y, Yan Y, Zhao J, Ding M, Zhang J, Jiang Y, Zhai Y and Duan Z: Comparative serum proteomic analysis of patients with acute-on-chronic liver failure: Alpha-1-acid glycoprotein maybe a candidate marker for prognosis of hepatitis B virus infection. J Viral Hepat 17: 816-824, 2010.

8. Yang L, Rudser KD, Higgins L, Rosen HR, Zaman A, Corless CL, David L and Gourley GR: Novel biomarker candidates to predict hepatic fibrosis in hepatitis $\mathrm{C}$ identified by serum proteomics. Dig Dis Sci 56: 3305-3315, 2011.

9. Jin GZ, Li Y, Cong WM, Yu H, Dong H, Shu H, Liu XH, Yan GQ, Zhang L, Zhang Y, et al: iTRAQ-2DLC-ESI-MS/MS based identification of a new set of immunohistochemical biomarkers for classification of dysplastic nodules and small hepatocellular carcinoma. J Proteome Res 10: 3418-3428, 2011.

10. Lee HJ, Na K, Choi EY, Kim KS, Kim H and Paik YK: Simple method for quantitative analysis of N-linked glycoproteins in hepatocellular carcinoma specimens. J Proteome Res 9: 308-318, 2010.

11. Goh WW, Lee YH, Zubaidah RM, Jin J, Dong D, Lin Q, Chung MC and Wong L: Network-based pipeline for analyzing MS data: An application toward liver cancer. J Proteome Res 10 2261-2272, 2011

12. DeSouza LV, Grigull J, Ghanny S, Dubé V, Romaschin AD, Colgan TJ and Siu KW: Endometrial carcinoma biomarker discovery and verification using differentially tagged clinical samples with multidimensional liquid chromatography and tandem mass spectrometry. Mol Cell Proteomics 6: 1170-1182, 2007.

13. Al Badaai Y, DiFalco MR, Tewfik MA and Samaha M: Quantitative proteomics of nasal mucus in chronic sinusitis with nasal polyposis. J Otolaryngol Head Neck Surg 38: 381-389, 2009.

14. Hergenroeder G, Redell JB, Moore AN, Dubinsky WP Funk RT, Crommett J, Clifton GL, Levine R, Valadka A and Dash PK: Identification of serum biomarkers in brain-injured adults: Potential for predicting elevated intracranial pressure. J Neurotrauma 25: 79-93, 2008.

15. Charlton M, Viker K, Krishnan A, Sanderson S, Veldt B, Kaalsbeek AJ, Kendrick M, Thompson G, Que F, Swain J, et al: Differential expression of lumican and fatty acid binding protein-1: New insights into the histologic spectrum of nonalcoholic fatty liver disease. Hepatology 49: 1375-1384, 2009.

16. Niu D, Sui J, Zhang J, Feng H and Chen WN: iTRAQ-coupled 2-D LC-MS/MS analysis of protein profile associated with HBV-modulated DNA methylation. Proteomics 9: 3856-3868, 2009

17. Feng H, Li X, Niu D and Chen WN: Protein profile in HBx transfected cells: A comparative iTRAQ-coupled 2D LC-MS/MS analysis. J Proteomics 73: 1421-1432, 2010.
18. Nayak D, Huo Y, Kwang WX, Pushparaj PN, Kumar SD, Ling EA and Dheen ST: Sphingosine kinase 1 regulates the expression of proinflammatory cytokines and nitric oxide in activated microglia. Neuroscience 166: 132-144, 2010.

19. Bantscheff M, Schirle M, Sweetman G, Rick J and Kuster B: Quantitative mass spectrometry in proteomics: A critical review. Anal Bioanal Chem 389: 1017-1031, 2007.

20. Li X, Hu B, Ding J and Chen H: Rapid characterization of complex viscous samples at molecular levels by neutral desorption extractive electrospray ionization mass spectrometry. Nat Protoc 6: 1010-1025, 2011.

21. Couttas TA, Raftery MJ, Erce MA and Wilkins MR: Monitoring cytoplasmic protein complexes with blue native gel electrophoresis and stable isotope labelling with amino acids in cell culture: Analysis of changes in the 20S proteasome. Electrophoresis 32: 1819-1823, 2011.

22. Albaum SP, Hahne H, Otto A, Haußmann U, Becher D, Poetsch A, Goesmann A and Nattkemper TW: A guide through the computational analysis of isotope-labeled mass spectrometry-based quantitative proteomics data: An application study. Proteome Sci 9: 30, 2011.

23. Sassek M, Pruszynska-Oszmalek E, Nowacka-Woszuk J, Szczerbal I, Szczepankiewicz D, Kaczmarek P, Kolodziejski PA, Switonski M and Mackowiak P: Resistin - from gene expression to development of diabetes. J Biol Regul Homeost Agents 27: 647-654, 2013.

24. Li Y, Ding L, Hassan W, Abdelkader D and Shang J: Adipokines and hepatic insulin resistance. J Diabetes Res 2013: 170532, 2013.

25. Leandro-García LJ, Leskelä S, Landa I, Montero-Conde C, López-Jiménez E, Letón R, Cascón A, Robledo $\mathrm{M}$ and Rodríguez-Antona C: Tumoral and tissue-specific expression of the major human beta-tubulin isotypes. Cytoskeleton 67: 214-223, 2010.

26. Tegze B, Szállási Z, Haltrich I, Pénzváltó Z, Tóth Z, Likó I and Gyorffy B: Parallel evolution under chemotherapy pressure in 29 breast cancer cell lines results in dissimilar mechanisms of resistance. PLoS One 7: e30804, 2012.

27. Wheeler HE, Wing C, Delaney SM, Komatsu M and Dolan ME: Modeling chemotherapeutic neurotoxicity with human induced pluripotent stem cell-derived neuronal cells. PLoS One 10: e0118020, 2015.

28. Dhananjayan SC, Ramamoorthy S, Khan OY, Ismail A, Sun J, Slingerland J, O'Malley BW and Nawaz Z: WW domain binding protein-2, an E6-associated protein interacting protein, acts as a coactivator of estrogen and progesterone receptors. Mol Endocrinol 20: 2343-2354,2006.

29. Perrier J, Durand A, Giardina T and Puigserver A: Catabolism of intracellular N-terminal acetylated proteins: Involvement of acylpeptide hydrolase and acylase. Biochimie 87: 673-685, 2005.

30. Arnesen T, Van Damme P, Polevoda B, Helsens K, Evjenth R, Colaert N, Varhaug JE, Vandekerckhove J, Lillehaug JR, Sherman F, et al: Proteomics analyses reveal the evolutionary conservation and divergence of N-terminal acetyltransferases from yeast and humans. Proc Natl Acad Sci USA 106: 8157-8162, 2009.

31. Goetze S, Qeli E, Mosimann C, Staes A, Gerrits B, Roschitzki B Mohanty S, Niederer EM, Laczko E, Timmerman E, et al: Identification and functional characterization of N-terminally acetylated proteins in Drosophila melanogaster. PLoS Biol 7: e1000236, 2009.

32. Kouzarides T: Acetylation: A regulatory modification to rival phosphorylation? EMBO J 19: 1176-1179, 2000.

33. Palmieri G, Bergamo P, Luini A, Ruvo M, Gogliettino M, Langella E, Saviano M, Hegde RN, Sandomenico A and Rossi M: Acylpeptide hydrolase inhibition as targeted strategy to induce proteasomal down-regulation. PLoS One 6: e25888, 2011. 